\title{
Adjuvant high-dose-rate brachytherapy in the management of oral cavity cancers: 5 years of experience in Iran
}

\author{
Ali Kazemian, MD', Mohammad Babaei, MD', Marzieh Lashkari, MD', Reza Ghalehtaki, MD', Ata Garajei, MD², \\ Maziar Motiee-Langroudi, MD33, Ahmadreza Sebzari, MD4, Ramin Jaberi, PhDl, Soraya Gholami, BSc', \\ Somayyeh Babalovi, PhD 5 , Mahdi Aghili, MD' \\ IRadiation Oncology Research Center (RORC), Cancer Institute, Tehran University of Medical Sciences, Tehran, ${ }^{2}$ Department of Head and \\ Neck Surgical Oncology and Reconstructive Surgery, Cancer Institute, Tehran University of Medical Sciences, Tehran, ${ }^{3}$ Otorhinolaryngology \\ Research Center, Otolaryngology Department, Tehran University of Medical Sciences, Tehran, ${ }^{4}$ Cellular and Molecular Research Center. \\ Birjand University of Medical Sciences, Birjnad, ${ }^{5}$ Department of Medical Physics and Biomedical Engineering, Tehran University of Medical \\ Sciences, Tehran, Iran
}

\begin{abstract}
Purpose: Brachytherapy is a cost-effective method for the management of oral cavity cancers in low to middle income countries. We aimed to evaluate the clinical outcomes of high-dose-rate interstitial brachytherapy (HDR-IBT) in patients with oral cavity cancer.

Material and methods: From 2009 to 2013, 78 patients (49 combined external beam radiotherapy [EBRT] plus IBT and 29 IBT monotherapy) with oral cavity cancers had been treated in our center. Slightly more than half the patients were male, and the median age was 54 years. The treatment was planned based on the Paris system. The main outcomes were disease-free and overall survival.

Results: The median follow-up duration was 36.5 months (range, 1.17-54.23). The actuarial four-year overall and disease-free survival rates were $83 \%$ and $65 \%$, respectively. The local and locoregional control was achieved among $89.74 \%$ and $87.17 \%$ of patients, respectively. None of the factors including tumor size, node status, gender, and radiation modality (IBT alone vs. IBT + EBRT) had a significant statistical correlation to the local control rate. All the patients tolerated the planned treatment in the IBT alone group. Late complications included a case of trismus and three cases of catheter insertion site fibrosis.

Conclusions: HDR-IBT as a monotherapy or in combination with EBRT is an appropriate option for the management of oral cavity squamous cell carcinomas, and supports the improvement in treatment outcomes and toxicity profiles in adjuvant settings.

Key words: brachytherapy, high-dose-rate, oral cancer, oral cavity.

\section{Purpose}

Oral cavity cancer is the sixth common cancer worldwide, while it ranks third in Iran. The prevalence of this cancer is much higher in the developing countries, whereas there is scarce information regarding disease outcomes. Concluding from previous research, oral cavity cancer in Iran is typically seen as squamous cell carcinoma in male smokers in their fifties or sixties; it occurs in the tongue, buccal mucosa, or gingiva with about $30 \%$ chance of patient surviving for five years on an average $[1,2,3,4,5]$. This relatively poor outcome is partly due to diagnosis at late stages and ineffective treatment strategies in the past.
Oral cavity cancer is a true example of the role of multiple medical armamentaria against cancer, including surgery, radiotherapy (external or brachytherapy), and chemotherapy. Historically, surgical excision was the mainstay treatment in early lesions, and gradually, radiation and chemotherapy were added for survival benefits as an addition to surgery. Nowadays, no one can deny the role of radiotherapy as a part of the best therapeutic strategy in head and neck cancers, and even the sole use of radiotherapy shows similar results to surgery in early lesions [6]. After the establishment of clinical outcomes recognized by radiation in the treatment 
of oral cavity cancers, both short-term and long-term toxic effects of external beam radiotherapy (e.g., acute mucositis and xerostomia, respectively) made radiation oncologists seek for safer treatment delivery techniques. The advantage of brachytherapy is based upon the inverse square law rule; i.e., the radiation penetrance is inversely related to the root of distance, so, while covering the whole of the suspicious areas with brachytherapy, we can preserve a considerable volume of the normal surrounding tissues.

In the Iran Cancer Institute, surgical resection is the first step of the therapeutic protocol in oral cavity cancers, and after the resection, all patients are referred for consultation regarding whether they need adjuvant radiation. In this study, we aimed to assess the outcomes of high-dose-rate interstitial brachytherapy (HDR-IBT) as a monotherapy or a boost to three-dimensional conformal radiotherapy in oral cavity cancer patients.

\section{Material and methods}

\section{Patient characteristics}

We studied consecutive patients with pathologically proven squamous cell carcinoma of the oral cavity that were treated with adjuvant HDR-IBT. From 2009 to 2013, 78 patients with oral cavity cancers had been treated with HDR-IBT in our Center. Twenty-nine patients received HDR-IBT monotherapy, while 49 underwent combined modality radiation therapy. Of these, $56.4 \%$ were male and the median age was 54 years (range, 18-89). Of the different sites in the oral cavity, the malignant lesion was located on the tongue in 70 patients, on the floor of the mouth in four patients, on the lips in two patients, and on the buccal mucosa in two patients.

The indications for radiotherapy (brachytherapy boost) in adjuvant settings were positive or close surgical margins, T3/T4 primary tumors, young age, or node positive T1-T2 tumors. In the patients with node-negative T1-2 oral tongue tumors with deep muscle invasion and no other adverse features, HDR-IBT was selected as a monotherapy. The metastatic cases were excluded from our study. If patients had clinically positive nodes or high risks of occult metastases, the regional lymphatics were treated with conformal 3D external beam radiotherapy (3D-EBRT) combined with IBT. The median tumor size and thickness were $2 \mathrm{~cm}$ (mean, $2.2 \pm 1.26$; range, 0.3-7) and $10 \mathrm{~mm}$ (mean, $10.2 \pm 6.51$; range, 2-30), respectively. The pathologic TNM classification of tumors in our study is shown in Table 1.

Table 1. Pathologic tumor and node staging number and \% of total cases

\begin{tabular}{lcccc} 
& T1 & T2 & T3 & Total \\
\hline Node negative & $42(53.8 \%)$ & $14(17.9 \%)$ & $5(6.4 \%)$ & $61(78.2 \%)$ \\
\hline Node positive & $7(8.9 \%)$ & $8(10.2 \%)$ & $2(2.6 \%)$ & $17(21.8 \%)$ \\
\hline Total & $49(62.8 \%)$ & $22(28.2 \%)$ & $7(8.9 \%)$ & $78(100 \%)$
\end{tabular}

\section{Implantation technique}

There was a 1-2 week interval between the external beam radiation and IBT to deliver a boost dose to the primary lesion. When IBT alone was used, it was usually initiated 3-4 weeks after surgical resection, a time usually considered for patient recovery and pathologic review. All patients were hospitalized for the entire duration of HDR-IBT. Before the initiation of the procedure, the radiation oncologists defined the desired treatment target volume using clinical examination and pre-op imaging studies (magnetic resonance imaging [MRI] and computed tomography [CT] scans) in advance. The target in almost all cases was the tumor bed after a complete resection plus 1 to $2 \mathrm{~cm}$ margin according to the Paris system. Under general anesthesia, the implantation of the flexible catheters was performed through the ipsilateral submental and submandibular triangular region into the target, approximately $10 \mathrm{~mm}$ apart and parallel to each other in a volume implant manner (Figures 1). The patients received appropriate intravenous antibiotics ( $1^{\text {st }}$ generation cephalosporin) and steroids (dexamethasone) during and after the implant procedure to reduce the inflammatory reaction of the target tissue, leaving the simulation procedure more accurate.

\section{Treatment planning, dose prescription and treatment delivery}

The day after the implantation, all patients underwent non-contrast multislice CT simulation for the 3D brachytherapy plan. The CT scan sections were $1 \mathrm{~mm}$ thick and were obtained from the skull base to the base of the tongue. The treatment planning was performed by using the brachytherapy planning system from Flexiplan (Isodosecontrol BV., Veenendaal, The Netherlands). The surrounding area connecting the peripheral catheters, encompassing the tumor bed, plus at least a $5 \mathrm{~mm}$ margin, formed the clinical target volume (CTV). Additionally, the mandible was delineated as the main organ-at-risk to prevent osteoradionecrosis. The proposed plan by the medical physicists was controlled by the radiation oncologists focusing on $\mathrm{D}_{90 \%}$ (the dose that $90 \%$ of the PTV (planning target volume) volume receives), $\mathrm{D}_{99 \%}$ (the dose that $99 \%$ of PTV receives), $V_{150}$ (the area that receives $150 \%$ of the prescribed dose), $V_{200}$ (the area that receives $200 \%$ of the prescribed dose, that should ideally be less than $20 \%$ ). We also evaluated other recommended indices including conformity index $(100 \%$ was ideal, but $\geq 70 \%$ was acceptable), dose non-uniformity ratio (DNR), which was calculated as $\mathrm{V}_{150} / \mathrm{V}_{100}$ (the ideal value was less than 0.5 ), and homogeneity index (HI), which was defined as $\mathrm{V}_{100}-\mathrm{V}_{150}$ / $\mathrm{V}_{100}$ (a complement to DNR) (Table 2). Then, irradiation was performed by connecting the catheters to our afterloading machine (Flexitron, Nucletron, an Elekta company, Elekta AB, Stockholm, Sweden). This device uses ${ }^{192}$ Ir stepping source, which is transferred to the desired catheters for different dwell times. All of the treatments were performed by using the HDR-IBT afterloading device with ${ }^{192} \mathrm{Ir}$ source in the strength range between 0.02 and $0.17 \mathrm{cGy} / \mathrm{m}^{2}$ of total reference air kerma (TRAK) per fraction. 
A
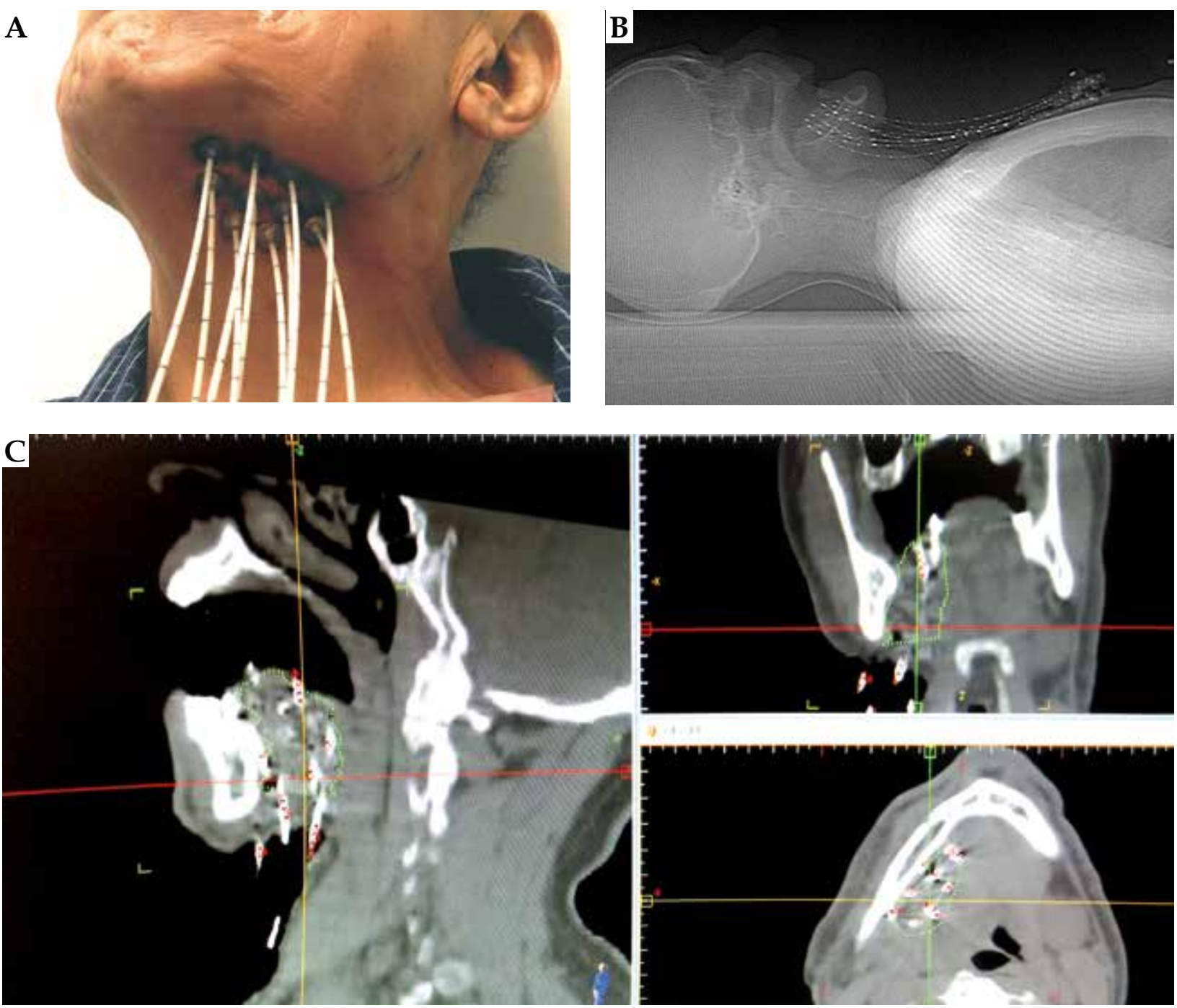

Fig. 1. The patient underwent adjuvant high-dose-rate brachytherapy for oral tongue carcinoma. A) Catheters inserted through submandibular region. B) The position of catheters in sagittal view of digital reconstruction radiograph obtained with computed tomography simulation. C) The position of catheters in sagittal, coronal, and axial views in treatment planning system

Two different dose schedules were selected, based on the radiation modality being used. In the patients who received IBT alone, the total dose was $39 \mathrm{~Gy}$, in $3 \mathrm{~Gy}$ fractions bid/ five days a week, with a six-hour interval between the fractions. In addition, IBT was combined with EBRT among the patients with locally-advanced disease (T3/4 or node-positive) who received 15-16 Gy in 3-4 Gy-fractions bid, usually after 45-50 Gy of EBRT in daily 2 Gy-fractions. The mean prescribed dose for the adjuvant and salvage treatment is presented in Table 3. The median dose of brachytherapy was 39 Gy (BED = 50.7 Gy) and 15 Gy (BED = 19.5 Gy) in the exclusive IBT and the combined IBT+EBRT groups, respectively. The median dose of EBRT was 46 Gy in the combined group.

The mean number of catheters used to achieve the optimal dose distribution was $9.6 \pm 2.65$. The mean clinical tar-

Table 2. Brachytherapy planning parameters - mean and standard deviation

\begin{tabular}{|c|c|c|c|c|c|c|c|}
\hline & $\begin{array}{c}D_{90} \\
(\% \text { of PD) }\end{array}$ & $\begin{array}{c}\mathrm{D}_{99} \\
(\% \text { of } \mathrm{PD})\end{array}$ & $\begin{array}{c}\mathrm{V}_{100} \\
\text { (\% of CTV) }\end{array}$ & $\begin{array}{c}\mathrm{V}_{150} \\
(\% \text { of CTV) }\end{array}$ & $\begin{array}{c}V_{200} \\
\text { (\% of CTV) }\end{array}$ & DNR & $\mathrm{HI}$ \\
\hline Combined BT \& EBRT & $\begin{array}{c}2.9 \pm 2.6 \\
(96.8 \%)\end{array}$ & $\begin{array}{l}2.7 \pm 3.9 \\
(68.7 \%)\end{array}$ & $\begin{array}{c}23.7 \pm 1.4 \\
(87.6 \%)\end{array}$ & $\begin{array}{c}11.6 \pm 5.1 \\
(44.7 \%)\end{array}$ & $\begin{array}{c}5.7 \pm 9.2 \\
(16.7 \%)\end{array}$ & $0.51 \pm 0.07$ & $0.49 \pm 0.07$ \\
\hline Exclusive BT & $\begin{array}{l}4.5 \pm 7.2 \\
(98.9 \%)\end{array}$ & $\begin{array}{c}3.4 \pm 5.2 \\
(74.2 \%)\end{array}$ & $\begin{array}{c}28.7 \pm 10.8 \\
(89.2 \%)\end{array}$ & $\begin{array}{c}13.9 \pm 5.9 \\
(43.3 \%)\end{array}$ & $\begin{array}{c}4.8 \pm 2.8 \\
(14.9 \%)\end{array}$ & $0.48 \pm 0.07$ & $0.51 \pm 0.07$ \\
\hline
\end{tabular}

BT - brachytherapy, EBRT - external beam radiotherapy, $D_{90}$. $D_{99}$ - the minimum dose to $90 \%, 99 \%$ of the CTV, $V_{100 \%}, V_{150 \%}, V_{200 \%}$ - volume of the anatomic volume receiving $100 \%, 150 \%, 200 \%$ of the prescribed dose, $P D$ - prescribed dose, DNR - dose non-uniformity ratio, $\mathrm{HI}$ - homogeneity index 
Table 3. Dose prescription - mean, standard deviation, dose range (minimum-maximum)

\begin{tabular}{lccccc} 
& BT dose & BED & EQD $_{2}$ & EBRT dose & Total dose (2 Gy/fr.) \\
\hline Combined BT \& EBRT $(n=51)$ & $17 \pm 7.84$ & $21.9 \pm 10.43$ & $18.2 \pm 8.7$ & $46 \pm 10.20$ & $64.4 \pm 5.87$ \\
& $(9-40)$ & $(11.7-56)$ & $(9.75-46.7)$ & $(14-68)$ & $(53-81.2)$ \\
\hline Exclusive BT $(n=27)$ & $40 \pm 1.67$ & $51.4 \pm 2.17$ & $42.9 \pm 1.8$ & & \\
& $(39-45)$ & $(50.7-58.5)$ & $(42.2-48.7)$ & & $64.4 \pm 5.87$ \\
\hline Total $(n=78)$ & $25 \pm 12.67$ & $32.1 \pm 16.51$ & $26.7 \pm 13.8$ & $46 \pm 10.20$ & $(53-81.2)$
\end{tabular}

$B T$ - brachytherapy, EBRT-external beam radiotherapy, fr. - fraction, $B E D$ - biological effective dose based on $\alpha / \beta=10$ for head-and-neck cancer, EQD $2-$ equivalent dose for 2 Gy-fraction treatment

get volume was $26.4 \pm 11.3$ and $32.3 \pm 12.8 \mathrm{ml}$ in combined BT + EBRT and BT alone, respectively. Other brachytherapy planning parameters are presented in Table 2.

\section{Peri-radiotherapy care and follow-up}

Deontological consultation was mandatory before the initiation of the treatment, and any intervention was performed about 10 days prior to the first fraction. The patients, during IBT, were visited daily in the admissions ward, whereas during EBRT were seen weekly in the clinic to monitor any possible treatment-related toxicity. After the completion of radiotherapy, the patients were told to return a month later for the first follow-up visit. The routine follow-up visits were made every three months for two years, except for the patients who confronted a problem requiring urgent assessment. The patients were evaluated both clinically (for catheter site necrosis, fibrosis, osteoradionecrosis, and xerostomia) and radiologically (ultrasonography - US) every three months and by MRI every six months for the first two years. Then, they were evaluated by US every six months and with an annual MRI for up to five years. When there was a suspicion over a locoregional recurrence, fine needle aspiration (FNA) or core needle biopsy was called for. In case of a confirmed recurrence, a surgical consultation was completed to assess the resectability of the disease, and when unresectable, radioand/or chemotherapy were the expected options.

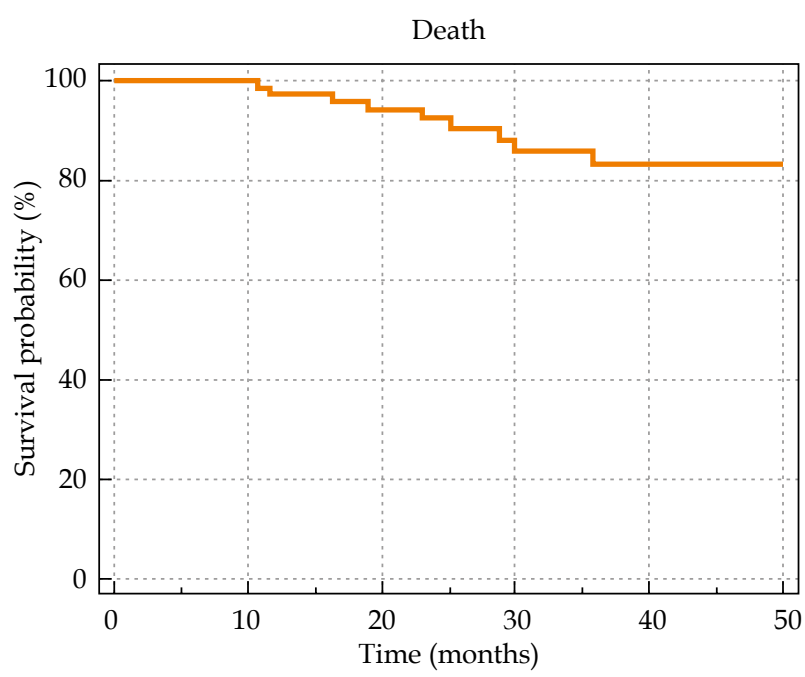

Fig. 2. Overall survival based on treatment setting.

\section{Statistical analysis}

The time interval between the last fraction of radiotherapy to the last uneventful follow-up or death due to any reason was considered for the calculation of overall survival. Similarly, disease free survival was defined as the time between end of radiotherapy to last uneventful visit, or occurrence of failure or death. All statistical analyses were performed by using the software SPSS version 20 (IBM Corp., Chicago, IL, USA). The actuarial survival rate and curves were estimated according to the KaplanMeier method.

\section{Results}

\section{Clinical outcomes}

Local control and survival

The median follow-up duration was 36.5 months (range, 1.17-54.23). Nine patients died during the follow-up period of their disease. The actuarial 4-year overall and disease-free survival rates were $83 \%$ and $65 \%$, respectively (Figures 2 and 3). The local and locoregional control were achieved among $89.74 \%$ and $87.17 \%$ of the patients, respectively. The locoregional control rate was $81.4 \%$ among those treated with IBT alone versus $90 \%$ among those treated the combined IBT-EBRT. None of the factors including tumor size, node status, gender, and

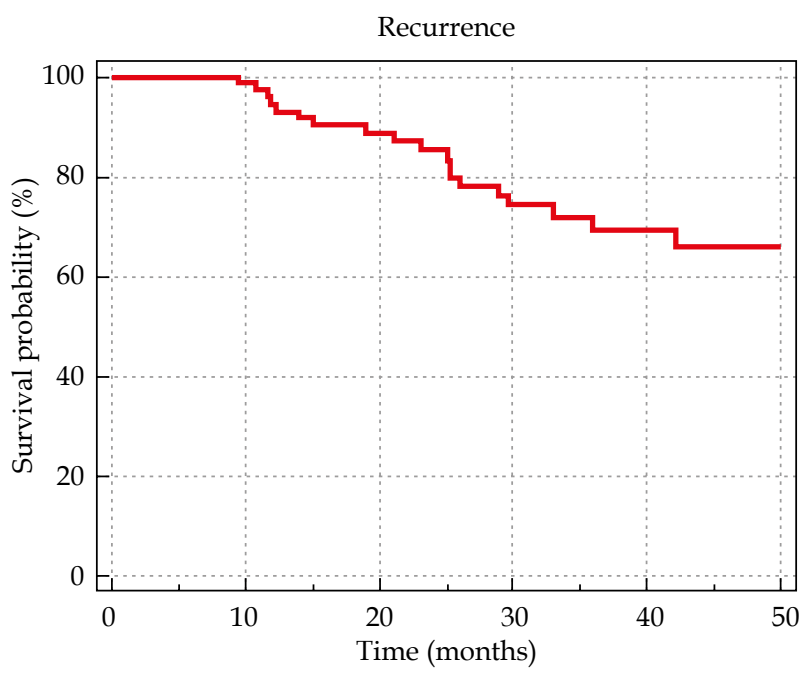

Fig. 3. Disease free survival based on treatment setting. 
radiation modality (IBT alone vs. IBT + EBRT) had a significant statistical correlation with the local control rate.

In five patients who were treated with IBT monotherapy, the cancer recurred (one in the primary site and four in the neck). The time interval between the brachytherapy and the local and neck recurrence were 12 and 18.1 months, respectively. Interestingly, no distant recurrences occurred. Our patient with local recurrence had a node negative $\mathrm{T} 2$ tongue tumor, whereas the regional recurrent cases occurred in patients with $\mathrm{T} 1$ (2 patients) and T2 (2 patients) tumors of the tongue. After performing neck dissection for two of the four regional recurrent cases, pathologic examinations revealed tumor cells seeding in the cervical soft tissue and not lymphatic metastasis.

In the combined modality group, five out of 51 patients reported locoregional recurrence. Considering the recurrence sites, four occurred in the oral cavity (local) and one in the neck (regional). The mean time to local and regional recurrences was $12 \pm 6.3$ and $12 \pm 9.5$ months, respectively. Distant metastasis occurred in two patients.

\section{Treatment-related complications}

All patients tolerated the planned treatment in the IBT alone group. There were no treatment-related acute complications that led to interruptions. Nevertheless, there were some interruptions due to acute toxic effects of external beam radiation, but no patient left without being subjected to the total prescribed dose. However, one patient complained of trismus. He was 54 years of age and suffered from a pT2N0 tongue squamous cell carcinoma (SCC) that underwent adjuvant combined IBT + EBRT (total BED, 65.5 Gy). There were no reports of osteoradionecrosis (ORN) in our patients.

Among the patients treated by IBT alone and combined EBRT+IBT, the rate of xerostomia was $2 / 27$ and $12 / 51$, respectively. Of two cases in the IBT monotherapy group, the first was a 54-year-old man with pT2N0 SCC of the tongue, and the second was a 57-year-old man with pT1N0 (tumor thickness, $10 \mathrm{~mm}$ ) SCC of the mouth floor. Both patients received 39 Gy in 13 fractions (BE, 50.7 Gy) after surgical resection with clear margins. Their problem persisted up to their last follow-up visit. The other 25 patients in the IBT alone group did not complain of xerostomia. Among the other studied complications, there were three cases of catheter insertion site fibrosis, including two in the combined and one in the IBT alone group. The patient who received only IBT (39 Gy/13 fractions) was a 54-year-old man with pT2N0 tongue tumor. Therefore, the total late complication rate (other than xerostomia) was $3.84 \%(3 / 78)$ in our patients.

\section{Discussion}

Surgery, radiotherapy, chemotherapy, or combined modalities are general treatment options for patients with oral cavity cancers. The choice of treatment modality depends on the stage of the tumor, the toxicity of the treatment, and patients comorbidities. Single modality treatment is preferred for the early stages of T1-T2 tumors. Although the outcome of radiation is equal to surgery in terms of local control, surgical excision is the preferred initial approach for early stage oral cavity cancers. After the surgery, adjuvant radiotherapy for improving local control is considered for patients with adverse pathologic features, i.e., neck lymph node involvement, perineural invasion, close margin, poorly differentiated tumor, or deep muscle invasion of oral tongue cancer.

In patients who are candidates for adjuvant external radiotherapy, especially with the oral tongue, mandible, and salivary glands as the irradiated fields, xerostomia and caries are the undesirable side effects of the treatment. For patients with high-risk local failure except regional recurrence, adjuvant radiotherapy could be performed by brachytherapy as a whole or as a boost to external radiotherapy. Brachytherapy is the best way to irradiate tumor bed and encompass contiguous spread of the disease while preserving healthy tissues. Hence, it is associated with lesser side effects compared to EBRT. The advantages of brachytherapy in head and neck cancer consist of a very local irradiation due to inverse square law, a high gradient dose around the tumor and a rapid dose fall-off around the radioactive sources, making it possible to increase the tumor-received dose and sparing the surrounding structures (such as tempro-mandibular joint (TMJ), salivary gland, mandible). Short duration of overall treatment reduces the risk of tumor repopulation, decreases integral dose, and presents the best dose conformity for the tumor.

Since the introduction of the Paris system for interstitial brachytherapy in the 1960s, this modality, due to its promising results, has gained a great deal of popularity in the management of patients with early stages of oral cavity cancers [7]. However, there has not been a head-to-head comparison of surgery versus radiotherapy $(\mathrm{BRT} \pm \mathrm{EBRT})$ as the primary treatment modalities so far, though, the outcomes have been comparable $[8,9]$. Thus, the choice of treatment should be completed on age, performance status, and quality of life [10]. Accordingly, reports suggest that definitive treatment with brachytherapy is not only safe, but also effective even in people above 80 -years of age when surgery is usually not possible $[11,12]$.

Our findings suggest that adjuvant brachytherapy (as a monotherapy or combined with EBRT) when added to surgery is effective in the treatment of oral cavity cancers. Using brachytherapy (completely or partially) instead of external beam radiation, when indicated, shortens the treatment time and protects the tissues from being exposed to radiation. These benefits are translated to decreased overall treatment time and reduction in machine overload rate, which is a major issue in developing countries with long waiting lists.

Interestingly, our results have shown that the fouryear overall survival of our patients exceeds $80 \%$. In addition, the disease-free survival rates were also encouraging. Another encouraging finding of this study was the extremely low rates of radiation-induced complications, especially xerostomia that lasts for a long time and is immune to treatment [13]. If anything, low complications should complement the large benefits seen with HDR-IBT. There are some explanations other than the use of BT for relatively high survival rates compared to similar reports 
in Iran and other Asian countries [1,5]. Based on earlier national reports, more than $50 \%$ of patients had stage III or IV [14], while in our series, the majority of the tumors were node-negative T1-T2, which implies better outcomes than more advanced lesions $[15,16,17]$. Another explanation is that all lesions were treated with surgery and postop radiation, which brings about better survival in comparison to surgery or definitive radiation alone $[18,19]$.

There are a number of reports in the literature presenting the efficacy of BT in primary malignant lesions of oral cavity. Most published studies show the use of low-dose-rate (LDR)-IBT. The incidence of long-term complications is significant, and necrosis and other undesirable side effects led to the discarding of this technique in most centers. Nowadays, HDR is becoming the standard in brachytherapy, but there is a fear of increasing late complications. Guinot et al., in patients with stage I/II oral tongue cancer, reported five years of disease-free survival of $79 \%$ patients who had undergone HDR-IBT. The dose of 40 Gy and 18 Gy were prescribed to patients when BT was used as monotherapy or boosted to 50 Gy EBRT, respectively [20]. In another study on primary mobile tongue cancer after partial glossectomy and BT monotherapy, Goineau et al. reported five-year local control and five-year overall survival of $76 \%$ and $56 \%$ in the patients with LDR-BT, respectively [21]. Kakimoto et al. indicated $71 \%$ three-year local control in T3 mobile tongue cancers with definitive HDR-BT [22]. In patients with definitive HDR-IBT, Lee et al. showed five-year overall survival and three-year local control of $70 \%$ and $84 \%$, respectively [23]. As evident, our outcomes are in agreement with the other reports despite the extensive variety in patient characteristics and treatment composition of surgery, external radiotherapy, and brachytherapy.

The first reports of HDR BT were performed with a limited number of patients and large dose fractions. The studies of Leung et al., Inoue et al., Lau et al., Umeda et al., and Yamazaki et al. were contradictory [24,25,26, $27,28,29,30,31]$. In these retrospective studies, the dose fractions of 3-6.5 Gy/fraction were used. Some of them presented that HDR would have the same local control rate with LDR, whereas others reported lower rates. Meanwhile, all of them consistently suggested more late adverse effects with HDR in comparison to LDR. Although the optimal dose fraction of HDR has not yet been defined, nowadays, after the accumulation of radiobiological experiences, it has been elucidated that an application of HDR-BT in fraction of 3 to 4 Gy is not more toxic than LDR in terms of late mucosal and soft tissue necrosis [32,33]. Thus, this dose per fraction was adopted by many institutions as the standard for HDR-BT schedules. In this study, we have no osteoradionecrosis, and only a limited number of soft tissue necrosis including re-irradiated patients. We considered dose optimization to avoid hyper dose sleeve $\left(D_{200 \%}\right)$ less than $14 \%$ of the irradiated volume and paid close attention to the mandible in order to avoid osteoradionecrosis. However, with this dose fraction, we still witnessed a grade 4 complication rate of about $5 \%$, which is relatively lower than the other reports in this context. For instance, the reported rates are $16 \%$ soft tissue necrosis by Guinot et al., 16\% grade
$3 / 4$ late complication by Hepel et al., and $22 \%$ of grade $\geq 2$ necrosis by Goineau et al. $[20,21,34]$. This difference in the frequency of complications is partly because we only reported grade 4 toxicity that required intensive intervention. Another explanation is that surgery and external radiotherapy, aside from $\mathrm{BT}$, have a major impact on the rate of complications, while these modalities have various contributions in the mentioned studies.

Our study had a number of limitations. The follow-up time was relatively short. The retrospective design of the study could impact the cause-effect relationship and limit our understanding of the predictors of survival. Many patients have been referred to our institution from far sites of the country, and this issue has negatively affected our follow-up duration. Some patients were referred to other centers after the occurrence of complications such as bone and soft tissue necrosis because we were short of dental facilities in our Institution.

\section{Conclusions}

Adjuvant brachytherapy, combined with external beam radiation or as a monotherapy, is associated with promising outcomes as well as good safety profiles for oral cavity cancers treated at our Centre. Thus, we are encouraged to use brachytherapy in the treatment of oral cavity cancers in various settings. However, our experience is mainly in adjuvant settings and there is a substantial need to test the efficacy of HDR brachytherapy in the definitive setting.

\section{Disclosure}

Authors report no conflict of interest.

\section{References}

1. Krishna RVK, Mejia G, Roberts-Thomson K, Logan R. Epidemiology of oral cancer in Asia in the past decade - an update (2000-2012). Asian Pac J Cancer Prev 2013; 14: 5567-5577.

2. Sargeran K, Murtomaa H, Safavi SM et al. Survival after diagnosis of cancer of the oral cavity. Br J Oral Maxillofac Surg 2008; 46: 187-191.

3. Fahmy MS, Sadeghi A, Behmard S. Epidemiologic study of oral cancer in Fars Province, Iran. Community Dent Oral Epidemiol 1983; 11: 50-58.

4. Andisheh-Tadbir A, Mehrabani D, Heydari ST. Epidemiology of squamous cell carcinoma of the oral cavity in Iran. J Craniofac Surg 2008; 19: 1699-1702.

5. Razmpa E, Memari F, Naghibzadeh B. Epidemiologic and Clinicopathologic Characteristics of Tongue Cancer in Iranian Patients. Acta Med Iran 2011; 49: 44-48.

6. Corvò R. Evidence-based radiation oncology in head and neck squamous cell carcinoma. Radiother Oncol 2007; 85: 156-170.

7. Mazeron JJ, Noël G, Simon JM. Head and neck brachytherapy. Semin Radiat Oncol 2002; 12: 95-108.

8. Shibuya H, Hoshina M, Takeda $M$ et al. Brachytherapy for stage I \& II oral tongue cancer: An analysis of past cases focusing on control and complications. Int J Radiat Oncol Biol Phys 1993; 26: 51-58.

9. Nag S, Cano ER, Demanes DJ et al. The American Brachytherapy Society recommendations for high-dose-rate brachytherapy for head-and-neck carcinoma Int J Radiat Oncol Biol Phys 2001; 50: 1190-1198.

10. Nakashima T, Nakamura K, Shiratsuchi H et al. Clinical outcome of partial glossectomy or brachytherapy in early-stage 
tongue cancer. Nihon Jibiinkoka Gakkai Kaiho 2010; 113: 456-462 [Article in Japanese].

11. Yamazaki H, Yoshida $K$, Kotsuma $T$ et al. Age is not a limiting factor for brachytherapy for carcinoma of the node negative oral tongue in patients aged eighty or older. Radiat Oncol 2010; 5: 116

12. Khalilur R, Hayashi K, Shibuya H. Brachytherapy for tongue cancer in the very elderly is an alternative to external beam radiation. Br J Radiol 2011; 84: 747-749.

13. Ameri A, Heydarirad G, Rezaeizadeh H et al. Evaluation of Efficacy of an Herbal Compound on Dry Mouth in Patients with Head and Neck Cancers. A Randomized Clinical Trial. J Evid Based Complementary Altern Med 2015; 21: 30-33.

14. Sargeran K, Murtomaa H, Safavi SMR et al. Malignant oral tumors in Iran: ten-year analysis on patient and tumor characteristics of 1042 patients in Tehran. J Craniofac Surg 2006; 17: $1230-1233$

15. Kruaysawat W, Aekplakorn W, Chapman RS. Survival time and prognostic factors of oral cancer in Ubon Ratchathani Cancer Center. J Med Assoc Thailand Chotmaihet Thangphaet 2010; 93: 278-284.

16. Prieto I, Prieto A, Bravo M et al. Prognostic factors for cancer of the oral cavity. Quintessence Int (Berl) 2005; 36.

17. Bettendorf O, Piffkò J, Bànkfalvi A. Prognostic and predictive factors in oral squamous cell cancer: important tools for planning individual therapy? Oral Oncol 2004; 40: 110-119.

18. Sargeran K, Murtomaa H, Safavi SM et al. Survival after diagnosis of cancer of the oral cavity. Br J Oral Maxillofac Surg 2008; 46: 187-191.

19. Zelefsky MJ, Zimberg SH, Raben A et al. Brachytherapy for locally advanced and recurrent lymph node metastases. J Brachytherapy Int 1998; 14: 123-132.

20. Guinot JL, Santos M, Tortajada MI et al. Efficacy of highdose-rate interstitial brachytherapy in patients with oral tongue carcinoma. Brachytherapy 2010; 9: 227-234.

21. Goineau A, Piot B, Malard O et al. Postoperative interstitial brachytherapy for resectable squamous cell carcinoma of the tongue. Brachytherapy 2014; 14: 71-76.

22. Kakimoto $\mathrm{N}$, Inoue $\mathrm{T}$, Inoue $\mathrm{T}$ et al. Results of low- and highdose-rate interstitial brachytherapy for T3 mobile tongue cancer. Radiother Oncol 2003; 68: 123-128.

23. Lee SU, Cho KH, Moon SH et al. Clinical outcome of highdose-rate interstitial brachytherapy in patients with oral cavity cancer. Radiat Oncol 2014; 32: 238-246.

24. Yamazaki H, Inoue T, Yoshida K et al. Brachytherapy for Early Oral Tongue Cancer: Low Dose Rate to High Dose Rate. I Radiat Res 2003; 44: 37-40.

25. Umeda M, Komatsubara H, Nishimatsu N et al. High-dose rate interstitial brachytherapy for stage I-II tongue cancer. Oral Surg Oral Med Oral Pathol Oral Radiol Endod 2000; 90: 667-670.

26. Leung TW, Wong VY, Kwan $\mathrm{KH}$ et al. High dose rate brachytherapy for early stage oral tongue cancer. Head Neck 2002; 24: 274-281.

27. Leung TW, Wong VYW, Wong CM et al. High dose rate brachytherapy for carcinoma of the oral tongue. Int J Radiat Oncol Biol Phys 1997; 39: 1113-1120.

28. Leung TW, Wong VYW, Wong CM et al. Technical hints for high dose rate interstitial tongue brachytherapy. Clin Oncol 1998; 10: 231-236.

29. Lau HY, Hay JH, Flores AD et al. Seven fractions of twice daily high dose-rate brachytherapy for node-negative carcinoma of the mobile tongue results in loss of therapeutic ratio. Radiother Oncol 1996; 39: 15-18.

30. Inoue T, Yoshioka Y, Shimamoto S et al. Phase III trial of high- vs. low-dose-rate interstitial radiotherapy for early mobile tongue cancer. Int J Radiat Oncol Biol Phys 2001; 51: 171-175.
31. Inoue $\mathrm{T}$, Inoue $\mathrm{T}$, Teshima $\mathrm{T}$ et al. Phase III trial of high and low dose rate interstitial radiotherapy for early oral tongue cancer. Int J Radiat Oncol Biol Phys 1996; 36: 1201-1204.

32. Petera J, Matula P, Paluska P et al. High dose rate versus low dose rate brachytherapy in the treatment of tongue carcinoma - a radiobiological study. Neoplasma 2009; 56: 163-168.

33. Nose T, Peiffert D, Lapeyre $\mathrm{M}$ et al. Late effects of post-highdose-rate brachytherapy for oropharyngeal carcinoma: are they severer than post-low-dose-rate? Strahlenther Onkol 2004; 180: 776-782.

34. Hepel JT, Syed AMN, Puthawala A et al. Salvage high-doserate (HDR) brachytherapy for recurrent head-and-neck cancer. Int J Radiat Oncol Biol Phys 2005; 62: 1444-1450. 